MPP-2013-148

\title{
Superstring/Supergravity Mellin Correspondence in Grassmannian Formulation
}

\author{
Stephan Stieberger ${ }^{1}$ and Tomasz R. Taylor ${ }^{2}$ \\ ${ }^{1}$ Max-Planck-Institut für Physik \\ Werner-Heisenberg-Institut, 80805 München, Germany \\ ${ }^{2}$ Department of Physics \\ Northeastern University, Boston, MA 02115, USA
}

\begin{abstract}
We extend the recently established Mellin correspondence of supergravity and superstring amplitudes to the case of arbitrary helicity configurations. The amplitudes are discussed in the framework of Grassmannian varieties. We generalize Hodges' determinant to a function of two sets of independent coordinates and show that tree-level supergravity amplitudes can be obtained by contour integrations of both sets in separate Grassmannians while in superstring theory, one set of coordinates is identified with string vertex positions at the disk boundary and Mellin transformed into generalized hypergeometric functions of Mandelstam invariants.
\end{abstract}


We have recently established a correspondence between superstring disk amplitudes for the scattering of $N$ gauge bosons and tree-level $N$-graviton supergravity amplitudes, when the external particles are in the maximally helicity violating (MHV) configurations [1]. We showed that in both cases, the amplitudes can be obtained from a single generating function - the Hodges' determinant [2] - in the supergravity case by identifying its arguments with the helicity spinors and in the superstring case by identifying them with string vertex positions on a disk. Integrating over vertex positions amount to multiple Mellin transforms which convert kinematic variables of supergravity amplitudes into generalized hypergeometric functions of Mandelstam invariants, with the string tension introduced as an energy unit.

In this work, we extend Mellin correspondence to the case of arbitrary helicity configurations. In order to incorporate Yang-Mills building blocks of the amplitudes, we represent them as contour integrals in Grassmannian varieties [3]. We will use the Veronese map from $G(2, n) \rightarrow G(k, n)[4]$, which is equivalent to the connected prescription in Witten's twistor string theory [5, 6] and allows a "particle interpretation" of the integrals.

We begin by recalling some properties of $N$-graviton MHV amplitudes [1, 2, 7-10]. The graviton polarization tensor is defined with reference to two (gauge fixing) null vectors or respectively, two spinors $x$ and $y$ that can be normalized as $\langle x y\rangle=1$. In the MHV case, Hodges' determinant can be expressed in terms of the Mandelstam invariants $s_{i j}$ and the projective variables

$$
\sigma_{k}^{1} \equiv\langle k x\rangle, \quad \sigma_{k}^{2} \equiv\langle k y\rangle, \quad k=1,2, \ldots N .
$$

In order to write the amplitude, it is convenient to introduce two sets of $\mathbb{C P}^{1}$ "position" coordinates

$$
z_{k}=\frac{\sigma_{k}^{1}}{\sigma_{k}^{2}}, \quad z_{k}^{\prime}=\frac{1}{z_{k}}=\frac{\sigma_{k}^{2}}{\sigma_{k}^{1}} .
$$

Note that

$$
z_{i}-z_{j} \equiv z_{i j}=\frac{(i j)}{\sigma_{i}^{2} \sigma_{j}^{2}}, \quad z_{i}^{\prime}-z_{j}^{\prime} \equiv z_{i j}^{\prime}=\frac{(j i)}{\sigma_{i}^{1} \sigma_{j}^{1}},
$$

where the determinant

$$
(i j) \equiv\left|\begin{array}{cc}
\sigma_{i}^{1} & \sigma_{j}^{1} \\
\sigma_{i}^{2} & \sigma_{j}^{2}
\end{array}\right|=\langle i j\rangle .
$$

Written in terms of these variables, up to an overall sign, the $N$-graviton MHV amplitude 
becomes [1]

$$
A_{N}^{G}=\left(\prod_{n=1}^{N} \sigma_{n}^{1} \sigma_{n}^{2}\right)^{-2} \frac{1}{z_{i j} z_{j k} z_{k i}} \frac{1}{z_{r s}^{\prime} z_{s t}^{\prime} z_{t r}^{\prime}}|\Psi|_{i j k}^{r s t},
$$

where $\Psi$ is a $N \times N$ "weighted Laplacian" matrix [2, 10] with the elements

$$
\psi_{i j}= \begin{cases}\frac{s_{i j}}{z_{i j} z_{i j}^{\prime}} & \text { if } i \neq j, \\ -\sum_{n \neq i} \frac{s_{i n}}{z_{i n} z_{i n}^{\prime}} & \text { if } i=j\end{cases}
$$

and $|\Psi|_{i j k}^{r s t}$ denotes the minor determinant obtained after deleting three rows $i, j, k$ and three columns $r, s, t$.

The amplitude is invariant under $\mathrm{GL}(2, \mathbb{C})$ symmetry reflecting its independence of the choice of reference spinors $(x, y)$. The homogenous coordinates $\sigma_{k}^{1,2}$ transform as spinor components under the Lorentz subgroup SL $(2, \mathbb{C})$, while the position coordinates undergo Möbius transformations:

$$
z_{k} \rightarrow \frac{a z_{k}+b}{c z_{k}+d}
$$

Note that $z^{\prime}=1 / z$ transform respectively. The $\mathrm{GL}(2, \mathbb{C})$ invariance holds for the MHV amplitude evaluated on a momentum-conserving, on-shell kinematic configuration with

$$
\begin{aligned}
& \sum_{j=1}^{N} s_{i j}=0, \\
& \sum_{j=1}^{N} \frac{s_{i j}}{z_{i j}}=0,
\end{aligned}
$$

where the primes over sums denote omission $j \neq i$.

In order to discuss general helicity configurations in supergravity and in superstring theory, we introduce the density

$$
H_{N}(\sigma, \tilde{\sigma})=\left(\prod_{n=1}^{N} \sigma_{n}^{2} \tilde{\sigma}_{n}^{2}\right)^{-2} \frac{1}{z_{i j} z_{j k} z_{k i}} \frac{1}{\tilde{z}_{r s} \tilde{z}_{s t} \tilde{z}_{t r}}|X|_{i j k}^{r s t}
$$

where the $N \times N$ matrix $X$ is defined as

$$
\chi_{i j}= \begin{cases}\frac{s_{i j}}{z_{i j} \tilde{z}_{i j}} & \text { if } i \neq j, \\ -\sum_{n \neq i} \frac{s_{i n}}{z_{i n} \tilde{z}_{i n}} & \text { if } i=j .\end{cases}
$$


The above expression has the same form as the MHV amplitude (5), but now it is considered as a function two independent sets of coordinates:

$$
\sigma_{k}^{1,2}: z_{k}=\frac{\sigma_{k}^{1}}{\sigma_{k}^{2}}, \quad \tilde{\sigma}_{k}^{1,2}: \tilde{z}_{k}=\frac{\tilde{\sigma}_{k}^{1}}{\tilde{\sigma}_{k}^{2}} .
$$

Here again,

$$
z_{i j}=\frac{(i j)}{\sigma_{i}^{2} \sigma_{j}^{2}}, \quad \tilde{z}_{i j}=\frac{(\tilde{\imath} \tilde{\jmath})}{\tilde{\sigma}_{i}^{2} \tilde{\sigma}_{j}^{2}},
$$

however, unlike in Eqs. (3]4), these coordinates are not yet related to helicity spinors or other kinematic variables.

Both superstring and supergravity amplitudes contain Yang-Mills building blocks. The Kawai-Lewellen-Tye (KLT) construction [11] of supergravity amplitudes involves "gluing" two Yang Mills amplitudes with appropriate kernels while the open superstring amplitudes can be obtained by gluing Yang-Mills with certain world-sheet integrals [12 14]. Following this path, we will obtain (one) Yang-Mills block of $\mathrm{N}^{k-2} \mathrm{MHV}$ amplitudes by a contour integration over the Grassmannian variety $G(k, N)$, with the variables $\tilde{\sigma}$ (and $\tilde{z}$ ) identified as the base coordinates of the Veronese map $\left(\mathbb{C}^{2}\right)^{N} / \mathrm{GL}(2) \rightarrow G(k, N)[4]$. The remaining $\sigma$ (and $z$ ) variables will be associated to Grassmannian coordinates of the second Yang-Mills block or to string vertex positions. In either case, we need to show that the density $H_{N}(10)$ is invariant under a larger $\mathrm{SL}(2) \times \widetilde{\mathrm{SL}}(2)$ symmetry group acting on $\sigma$ and $\tilde{\sigma}$.

The Grassmannian integration over $\tilde{\sigma}$ involves delta function constraints relating these coordinates to kinematic variables in a way respecting momentum conservation [3]. Actually, an explicit $\delta\left(\sum p\right)$ factor can be always extracted by appropriate change of integration variables. These kinematic relations are also responsible for the Bern-Carrasco-Johansson (BCJ) relations [15] between partial amplitudes. In this context, Cachazo [16] showed that:

$$
\sum_{j=1}^{N} s_{i j} \frac{(\tilde{n} \tilde{\jmath})}{(\tilde{\imath} \tilde{\jmath})}=0
$$

which, together with Eq. (8), imply

$$
\sum_{j=1}^{N} \frac{s_{i j}}{\tilde{z}_{i j}}=0
$$

thus generalizing Eq. (9) to arbitrary helicity configurations. This relation can be used for our purpose, to show that under SL(2), the $X$ matrix elements (11) transform as

$$
\chi_{i j} \rightarrow\left(c z_{i}+d\right)\left(c z_{j}+d\right) \chi_{i j}
$$


thus in Eq. (10), the corresponding factor from the determinant is canceled by the factor supplied by $\left(z_{i j} z_{j k} z_{k i}\right)^{-1}\left(\prod_{n=1}^{N} \sigma_{n}^{2}\right)^{-2}$. Hence $H_{N}$ is invariant under $\mathrm{SL}(2)$ transformations.

In order to demonstrate $\widetilde{\mathrm{SL}}(2)$-invariance, we need to differentiate between supergravity and superstring cases. In supergravity, $\sigma$ and $z$ variables will be associated to their own Grassmannian variety, hence they will be constrained in the same way as $\tilde{z}$ in Eq. (15), and $\widetilde{\mathrm{SL}}(2)$-invariance follows by the same argument. In superstring disk amplitudes, the SL(2)-invariant density $H_{N}$ will be weighted by Koba-Nielsen factors and integrated over $N$ (ordered) $z$ variables now identified with string vertex positions on $\mathbb{P}^{1}$. The corresponding string "formfactor" integrals are constrained by the vanishing integral of the total derivative

$$
\frac{\partial}{\partial z_{i}} \prod_{1 \leq k<l \leq N}\left|z_{k l}\right|^{s_{k l}}=\sum_{j=1}^{N} \frac{s_{i j}}{z_{i j}} \prod_{1 \leq k<l \leq N}\left|z_{k l}\right|^{s_{k l}} .
$$

Integrations by parts resulting in such vanishing total derivative terms were used in Ref. [17] to derive BCJ relations for $N$-point disk integrals. The bottom line is that the relation

$$
\sum_{j=1}^{N} \frac{s_{i j}}{z_{i j}}=0
$$

can be used in both supergravity and superstring amplitudes to show that up to total derivatives, the density $H_{N}$ is invariant under $\widetilde{\mathrm{SL}}(2)$ transformations. Furthermore, BCJ relations can be used on both $\mathrm{SL}(2)$ and $\widetilde{\mathrm{SL}}(2)$ sides.

Now we can use $\mathrm{SL}(2) \times \widetilde{\mathrm{SL}}(2)$ invariance to make connections with the KLT formula 11] for gravity amplitudes and with the general formula for the disk amplitudes [13, 17]. Although this can be done for an arbitrary set of $i, j, k, r, s, t$ indices in Eq. (10), in order to streamline the argument, we choose $i=r=1, j=s=N-1, k=t=N$. Then, according to the matrix-tree theorem, the determinant is given by the sum of all forests consisting of three trees rooted at $1, N-1$ and $N$, with a combined number of $N-3$ edges, each of them bringing a $\chi_{i j}$ factor [10]. At this point, we go to a $\operatorname{SL}(2)$ reference frame with $\sigma_{N}^{2}=0$ $\left(z_{N} \rightarrow \infty\right)$ and to a $\widetilde{\mathrm{SL}}(2)$ reference frame with $\tilde{\sigma}_{N-1}^{2}=0\left(\tilde{z}_{N-1} \rightarrow \infty\right)$. In this way, we are left with single trees only, rooted at 1, i.e. all trees with $N-2$ vertices different from $N-1$ and $N$. Next, we partial fraction $\tilde{z}_{i j}$ denominators on the $\widetilde{\mathrm{SL}}(2)$ side, as described in Ref. [1], use BCJ relations on the $\mathrm{SL}(2)$ side and finally restore $\mathrm{SL}(2) \times \widetilde{\mathrm{SL}}(2)$ invariance by returning to a general frame. All this seems rather involved, so it is worth illustrating on some simple examples. 
For $N=4$, we begin with

$$
H_{4}(\sigma, \tilde{\sigma})=\frac{1}{(13)(14)(34)} \frac{1}{(\tilde{1} \tilde{3})(\tilde{1} \tilde{4})(\tilde{3} \tilde{4})} \frac{1}{\left(\sigma_{2}^{2}\right)^{2}\left(\tilde{\sigma}_{2}^{2}\right)^{2}} \frac{s_{12}}{z_{12} \tilde{z}_{12}}
$$

which, in the reference frame with $\sigma_{4}^{2}=\tilde{\sigma}_{3}^{2}=0$, becomes

$$
H_{4}(\sigma, \tilde{\sigma})=\frac{1}{\tilde{\sigma}_{2}^{2} \tilde{\sigma}_{3}^{1}(\tilde{1} \tilde{4})(\tilde{3} \tilde{4})} \frac{1}{\sigma_{2}^{2} \sigma_{4}^{1}(13)(34)} \frac{s_{12}}{(12)(\tilde{1} \tilde{2})} .
$$

After reverting to a general reference frame,

$$
H_{4}(\sigma, \tilde{\sigma})=\frac{1}{(\tilde{1} \tilde{2})(\tilde{2} \tilde{3})(\tilde{3} \tilde{4})(\tilde{4} \tilde{1})} s_{12} \frac{1}{(12)(24)(43)(31)} .
$$

Starting from $N=5$, partial fractioning and BCJ relations become very helpful. After partial fractioning, we obtain:

$$
H_{5}(\sigma, \tilde{\sigma})=\frac{1}{(14)(15)(45)} \frac{1}{(\tilde{1} \tilde{4})(\tilde{1} \tilde{5})(\tilde{4} \tilde{5})} \frac{1}{\left(\sigma_{2}^{2} \sigma_{3}^{2}\right)^{2}\left(\tilde{\sigma}_{2}^{2} \tilde{\sigma}_{3}^{2}\right)^{2}} \frac{1}{\tilde{z}_{12} \tilde{z}_{23}} \frac{s_{12}}{z_{12}}\left(\frac{s_{13}}{z_{13}}+\frac{s_{23}}{z_{23}}\right)+(2 \leftrightarrow 3) .
$$

BCJ relations are implemented by applying Eq. (18) to the bracket on the r.h.s. which, in the reference frame of $z_{5} \rightarrow \infty$, becomes:

$$
\frac{s_{13}}{z_{13}}+\frac{s_{23}}{z_{23}}=\frac{s_{34}}{z_{34}} .
$$

Staying in this frame, we obtain:

$$
H_{5}(\sigma, \tilde{\sigma})=\frac{1}{\tilde{\sigma}_{3}^{2} \tilde{\sigma}_{4}^{1}(\tilde{1} \tilde{5})(\tilde{4} \tilde{5})(\tilde{1} \tilde{2})(\tilde{2} \tilde{3})} \frac{s_{12} s_{34}}{\sigma_{2}^{2} \sigma_{5}^{1} \sigma_{3}^{2} \sigma_{5}^{1}(14)(12)(34)}+(2 \leftrightarrow 3)
$$

After reverting to a general reference frame, we obtain

$$
H_{5}(\sigma, \tilde{\sigma})=\widetilde{M}(1,2,3,4,5) s_{12} s_{34} M(2,1,4,3,5)+(2 \leftrightarrow 3)
$$

with the definitions

$$
\begin{aligned}
& M\left(i_{1}, i_{2}, \ldots, i_{N}\right)=\frac{1}{\left(i_{1} i_{2}\right)\left(i_{2} i_{3}\right) \cdots\left(i_{N} i_{1}\right)}, \\
& \widetilde{M}\left(i_{1}, i_{2}, \ldots, i_{N}\right)=\frac{1}{\left(\tilde{\imath}_{1} \tilde{\imath}_{2}\right)\left(\tilde{\imath}_{2} \tilde{\imath}_{3}\right) \cdots\left(\tilde{\imath}_{N} \tilde{\imath}_{1}\right)},
\end{aligned}
$$

which become useful for higher $N$.

The $N=4$ and $N=5$ examples should make it clear how to proceed to higher $N$. The result can be written in many ways, the shortest involving $(N-3) ! \times\left(\left\lfloor\frac{N}{2}\right\rfloor-1\right) ! \times\left(\left\lceil\frac{N}{2}\right\rceil-2\right) !$ terms 1 being:

\footnotetext{
$1\lfloor x\rfloor$ is the integer part of $x$, while $\lceil x\rceil$ gives the smallest integer greater than or equal to $x$.
} 


$$
\begin{aligned}
H_{N}(\sigma, \tilde{\sigma})= & \sum_{\pi \in S_{N-3}} \widetilde{M}(1, \pi(2,3, \ldots, N-2), N-1, N) \\
& \times \sum_{\alpha \in S_{\lfloor N / 2\rfloor-1}} \widetilde{S}(\alpha \circ \pi(2, \ldots,\lfloor N / 2\rfloor)) \sum_{\beta \in S_{\lceil N / 2\rceil-2}} S(\beta \circ \pi(\lfloor N / 2\rfloor+1, \ldots N-2)) \\
& \times M(\alpha \circ \pi(2, \ldots,\lfloor N / 2\rfloor), 1, N-1, \beta \circ \pi(\lfloor N / 2\rfloor+1, \ldots, N-2), N),
\end{aligned}
$$

where $\pi, \alpha$ and $\beta$ denote permutations of the respective numbers of $N-3,\lfloor N / 2\rfloor-1$ and $\lceil N / 2\rceil-2$ elements. Here,

$$
\begin{aligned}
& \widetilde{S}\left(i_{1}, \ldots, i_{j}\right)=s_{1 i_{j}} \prod_{m=1}^{j-1}\left(s_{1 i_{m}}+\sum_{k=m+1}^{j} \theta\left(i_{m}, i_{k}\right)\right) \\
& S\left(i_{1}, \ldots, i_{l}\right)=s_{i_{1} N-1} \prod_{m=2}^{l}\left(s_{i_{m} N-1}+\sum_{k=1}^{m-1} \theta\left(i_{k}, i_{m}\right)\right),
\end{aligned}
$$

with

$$
\theta(i, j)= \begin{cases}s_{i j}, & i>j \\ 0, & i<j\end{cases}
$$

are the elements of the KLT momentum kernel [8, 11, 18]. We conclude that the generalized Hodges' determinant density $H_{N}$ of Eq. (10) does indeed describe two Yang-Mills factors "glued" by KLT kernels. We will show below that it yields correct supergravity amplitudes and agrees with the general formula for open superstring amplitudes, when integrated with appropriate delta function constraints and Koba-Nielesen factors.

We are ready to apply the generalized Hodges' determinant as a pivot for connecting supergravity and superstring amplitudes in the Grassmannian framework. The tree level, $\mathrm{N}^{k-2} \mathrm{MHV}$ supergravity amplitude is given by

$$
\begin{aligned}
A_{k, N}^{G}= & \frac{1}{\operatorname{vol}(\mathrm{GL}(2)) \operatorname{vol}(\widetilde{\mathrm{GL}}(2))} \int d^{2} \sigma_{1} \cdots d^{2} \sigma_{N} \int d^{2} \tilde{\sigma}_{1} \cdots d^{2} \tilde{\sigma}_{N} H_{N}(\sigma, \tilde{\sigma}) \\
& \times \prod_{\alpha=1}^{k} \delta^{4 \mid 4}\left(\sum_{i=1}^{N} C_{\alpha i}^{V}[\sigma] \mathcal{W}_{i}(\eta)\right) \prod_{\tilde{\alpha}=1}^{k} \delta^{4 \mid 4}\left(\sum_{i=1}^{N} C_{\tilde{\alpha} i}^{V}[\tilde{\sigma}] \mathcal{W}_{i}(\tilde{\eta})\right),
\end{aligned}
$$

where $C^{V}$ is the Veronese map [4]. The kinematic data are specified by the (dual) $\mathcal{N}=4$ supertwistors $\mathcal{W}$. Note the presence of two sets of anticommuting variables, $\eta$ and $\tilde{\eta}$ which are necessary for the enhancement to $\mathcal{N}=8$ supersymmetry. Eq. (27) guarantees agreement with the KLT formula however it is easy to see that Eq. (30) overshoots by an extra $\delta\left(\sum p\right)$ 
factor - thus a pedantic reader should divide the r.h.s. by $\delta^{4}(0)$. We should note that the Grassmannian formulation of supergravity amplitudes and their twistor string origin have been considered before in Refs. [19, 20]; in particular, Ref. [19] makes a similar connection between Hodges' determinant and KLT formula.

The partial $\mathrm{N}^{k-2} \mathrm{MHV}$ open superstring amplitude is given, at the disk level, by

$$
\begin{aligned}
A_{k, N}^{S}= & \frac{1}{\operatorname{vol}(\operatorname{SL}(2)) \operatorname{vol}(\widetilde{\mathrm{GL}}(2))} \int_{D \subset\left(\mathbb{P}^{1}\right)^{N}} d^{2} \sigma_{1} \cdots d^{2} \sigma_{N} \int d^{2} \tilde{\sigma}_{1} \cdots d^{2} \tilde{\sigma}_{N} H_{N}(\sigma, \tilde{\sigma}) \\
& \times \prod_{1 \leq i<j \leq N}|(i j)|^{s_{i j}} \prod_{\tilde{\alpha}=1}^{k} \delta^{4 \mid 4}\left(\sum_{i=1}^{N} C_{\tilde{\alpha} i}^{V}[\tilde{\sigma}] \mathcal{W}_{i}(\tilde{\eta})\right),
\end{aligned}
$$

where the domain of $\sigma$-integration $D \subset\left(\mathbb{P}^{1}\right)^{N}$ is determined by color ordering. Eq. (27) guarantees agreement with the general formula for disk amplitudes [13], as recently recast in the KLT form in Ref. [17]. Note that the string integral, including the Koba-Nielsen factor $\prod_{1 \leq i<j \leq N}|(i j)|^{s_{i j}} \sim \prod_{1 \leq i<j \leq N}\left|z_{i j}\right|^{s_{i j}}$, is fully GL(2) invariant, but the integration domain is restricted to the disk boundary where string vertex positions are specified by $z$ coordinate 2 . When integrating on $\mathbb{P}^{1}$, we can set $\sigma_{i}^{2}=1$, so that $(i j)=z_{i j}$ and replace $d^{2} \sigma_{i} \rightarrow d z_{i}$. In Ref. [1], we argued that integrations with Koba-Nielsen factor amount to Mellin transforms with the measure

$$
\int d M_{N}=\frac{1}{\operatorname{vol}(\operatorname{SL}(2))} \int_{D \subset\left(\mathbb{P}^{1}\right)^{N}} d z_{1} \ldots d z_{N} \prod_{1 \leq i<j \leq N}\left|z_{i j}\right|^{s_{i j}}
$$

which are multi-dimensional Mellin transforms from the string world-sheet boundary to the Mellin space of Mandelstam invariants encoded in generalized hypergeometric functions. Thus Eqs. (30) and (31) extend the supergravity/superstring Mellin correspondence from the MHV case considered in Ref. [1] to all helicity configurations.

It is clear that the generalized Hodges' determinant (27) plays a central role in superstring/supergravity correspondence. This may indicate the existence of yet another, perhaps more fundamental theory. On the other hand, one could try to formulate Mellin correspondence without referring to this determinant. In principle, in order to construct string amplitudes, one could start from field-theoretical amplitudes or some motivic objects (cf. [21]) taken as functions of kinematic variables and consider transformations with respect to some

\footnotetext{
${ }^{2}$ Here, one also overshoots by an extra $\delta\left(\sum p\right)$ factor which in this case originates from the world-sheet correlator giving rise to the Koba-Nielsen factor.
} 
dual variables. This may be difficult though because there are many, often highly non-trivial but equivalent, ways of writing the amplitudes.

Field theory appears in the $\alpha^{\prime} \rightarrow 0$ limit of string theory. Going the other way, directly from field theory to full-fledged string theory by means of integral transforms is harder, but it may be possible with some better understanding of the amplitudes.

\section{Acknowledgements}

We thank Nima Arkani-Hamed, Sergio Ferrara and David Skinner for useful discussions and correspondence. Furthermore, we are grateful to the Theory Division of CERN for hospitality and financial support when finalizing this work. T.R.T. is grateful to Max-Planck-Institut für Physik in München for kind hospitality. This material is based in part upon work supported by the National Science Foundation under Grant No. PHY-0757959. Any opinions, findings, and conclusions or recommendations expressed in this material are those of the authors and do not necessarily reflect the views of the National Science Foundation.

[1] S. Stieberger and T.R. Taylor, "Superstring Amplitudes as a Mellin Transform of Supergravity," Nucl. Phys. B 873, 65 (2013) [arXiv:1303.1532 [hep-th]].

[2] A. Hodges, "A simple formula for gravitational MHV amplitudes," arXiv:1204.1930 [hep-th].

[3] N. Arkani-Hamed, F. Cachazo, C. Cheung and J. Kaplan, "A Duality For The S Matrix," JHEP 1003, 020 (2010) arXiv:0907.5418 [hep-th]].

[4] N. Arkani-Hamed, J. Bourjaily, F. Cachazo and J. Trnka "Unification of Residues and Grassmannian Dualities," JHEP 1101, 049 (2011) [arXiv:0912.4912 [hep-th]].

[5] E. Witten, "Perturbative gauge theory as a string theory in twistor space," Commun. Math. Phys. 252 (2004) 189 arXiv:hep-th/0312171].

[6] R. Roiban, M. Spradlin and A. Volovich, "On the tree level S matrix of Yang-Mills theory," Phys. Rev. D 70, 026009 (2004) hep-th/0403190.

[7] F.A. Berends, W.T. Giele and H. Kuijf, "On relations between multi - gluon and multigraviton scattering," Phys. Lett. B 211, 91 (1988). 
[8] Z. Bern, L.J. Dixon, M. Perelstein and J.S. Rozowsky, "Multileg one loop gravity amplitudes from gauge theory," Nucl. Phys. B 546, 423 (1999) hep-th/9811140.

[9] D. Nguyen, M. Spradlin, A. Volovich and C. Wen, "The Tree Formula for MHV Graviton Amplitudes," JHEP 1007, 045 (2010) arXiv:0907.2276 [hep-th]].

L.J. Mason and D. Skinner, "Gravity, Twistors and the MHV Formalism," Commun. Math. Phys. 294, 827 (2010) [arXiv:0808.3907 [hep-th]].

[10] B. Feng and S. He, "Graphs, determinants and gravity amplitudes," JHEP 1210, 121 (2012) arXiv:1207.3220 [hep-th]].

[11] H. Kawai, D.C. Lewellen and S.H.H. Tye, "A Relation Between Tree Amplitudes of Closed and Open Strings," Nucl. Phys. B 269, 1 (1986).

[12] D. Oprisa and S. Stieberger, "Six gluon open superstring disk amplitude, multiple hypergeometric series and Euler-Zagier sums," hep-th/0509042.

S. Stieberger and T.R. Taylor, "Multi-gluon scattering in open superstring theory," Phys. Rev. D 74 (2006) 126007 arXiv:hep-th/0609175].

S. Stieberger and T.R. Taylor, "Amplitude for N-gluon superstring scattering," Phys. Rev. Lett. 97 (2006) 211601 arXiv:hep-th/0607184.

S. Stieberger and T.R. Taylor, "Supersymmetry Relations and MHV Amplitudes in Superstring Theory," Nucl. Phys. B 793 (2008) 83 [arXiv:0708.0574 [hep-th]].

S. Stieberger and T.R. Taylor, "Complete Six-Gluon Disk Amplitude in Superstring Theory," Nucl. Phys. B 801, 128 (2008) [arXiv:0711.4354 [hep-th]].

[13] C.R. Mafra, O. Schlotterer and S. Stieberger, "Complete N-Point Superstring Disk Amplitude I. Pure Spinor Computation," Nucl. Phys. B 873, 419 (2013) [arXiv:1106.2645 [hep-th]]. C.R. Mafra, O. Schlotterer and S. Stieberger, "Complete N-Point Superstring Disk Amplitude II. Amplitude and Hypergeometric Function Structure,” Nucl. Phys. B 873, 461 (2013) arXiv:1106.2646 [hep-th]].

[14] S. Stieberger and T.R. Taylor, "Maximally Helicity Violating Disk Amplitudes, Twistors and Transcendental Integrals," Phys. Lett. B 716, 236 (2012) [arXiv:1204.3848 [hep-th]].

[15] Z. Bern, J. J. M. Carrasco and H. Johansson, "New Relations for Gauge-Theory Amplitudes," Phys. Rev. D 78, 085011 (2008) arXiv:0805.3993 [hep-ph]].

[16] F. Cachazo, "Fundamental BCJ Relation in N=4 SYM From The Connected Formulation," arXiv:1206.5970 [hep-th]. 
[17] J. Broedel, O. Schlotterer and S. Stieberger, "Polylogarithms, Multiple Zeta Values and Superstring Amplitudes," arXiv:1304.7267 [hep-th].

[18] N.E.J. Bjerrum-Bohr, P.H. Damgaard, T. Sondergaard and P. Vanhove, "The Momentum Kernel of Gauge and Gravity Theories," JHEP 1101 (2011) 001 [arXiv:1010.3933 [hep-th]].

[19] F. Cachazo and Y. Geyer, “A 'Twistor String' Inspired Formula For Tree-Level Scattering Amplitudes in N=8 SUGRA," arXiv:1206.6511 [hep-th].

[20] F. Cachazo and D. Skinner, "Gravity from Rational Curves," arXiv:1207.0741 [hep-th]. D. Skinner, "Twistor Strings for N=8 Supergravity," arXiv:1301.0868 [hep-th].

[21] O. Schlotterer and S. Stieberger, "Motivic Multiple Zeta Values and Superstring Amplitudes," arXiv:1205.1516 [hep-th]. 